\title{
CORRECTION
}

\section{Correction to: In vitro schistosomicidal activity of tamoxifen and its effectiveness in a murine model of schistosomiasis at a single dose}

\author{
Rosimeire N. Oliveira ${ }^{1,2} \cdot$ Sheila A. P. Corrêa ${ }^{1} \cdot$ Karen M. Vieira $^{1} \cdot$ Tiago Mendes $^{1} \cdot$ Silmara M. Allegretti $^{1}$ • \\ Danilo C. Miguel ${ }^{1}$
}

Published online: 22 March 2019

(C) Springer-Verlag GmbH Germany, part of Springer Nature 2019

\section{Correction to: Parasitology Research https://doi.org/10.1007/s00436-019-06259-0}

The original published version of this article contains error in

Tables 1 and 2. Correct tables are presented here.

Table 1 Worm recovery from the hepatic portal system and mesenteric intestinal veins of mice experimentally infected with $S$. mansoni and treated with PBS (control group) or one single dose of $100 \mathrm{mg} / \mathrm{kg}$ cTAM

\begin{tabular}{|c|c|c|c|c|c|c|}
\hline \multirow[t]{2}{*}{ Administration route } & \multirow[t]{2}{*}{ Treatment } & \multicolumn{5}{|c|}{ Warm burden (mean $\pm \mathrm{SD})$} \\
\hline & & Couples & Males & Females & Total & Total $\%$ reduction \\
\hline \multirow[t]{2}{*}{ Intrapentoneal } & Control & $8.5 \pm 3$ & $5.4 \pm 3.1$ & $3.9 \pm 1.4$ & $26.3 \pm 5.9$ & - \\
\hline & eTAM & $2.5 \pm 1^{*}$ & $1.3 \pm 0.9 * * *$ & $0.7 \pm 0.7$ & $7 \pm 2.3^{*}$ & $73 \%$ \\
\hline \multirow[t]{2}{*}{ Oral } & Control & $12.2 \pm 3.4$ & $5.8 \pm 1.9$ & $1.8 \pm 0.7$ & $32 \pm 6.4$ & - \\
\hline & eTAM & $6.7 \pm 1.9 * *$ & $0.9 \pm 1 * * * *$ & $0.5 \pm 0.6$ & $14.7 \pm 3.5^{*}$ & $54 \%$ \\
\hline
\end{tabular}

Significant reduction when compared with control group, $p<0.05$

$* p<0.001$

$* * p=0.0012$

$* * * p=0.021$

$* * * * p=0.0051$

The online version of the original article can be found at https://doi.org/ $10.1007 / \mathrm{s} 00436-019-06259-0$

Silmara M. Allegretti sallegre@unicamp.br

$\triangle$ Danilo C. Miguel dcmiguel@unicamp.br

1 Biology Institute, Department of Animal Biology - Parasitology, University of Campinas - UNICAMP, Campinas, São Paulo, Brazil

2 Present address: Biological and Health Sciences Section, Department of General Biology, Ponta Grossa State University, Ponta Grossa, Paraná, Brazil 
Table 2 Development singe of eggs retained in the intestinal tissues (oogram pattern); fecal egg count (EPG, number of eggs per gram of feces); and fecal egg count reduction (\%) of mice experimentally infected with $S$. mansoni and treated with PBS (control group) or one single dose of $100 \mathrm{mg} / \mathrm{kg}$ cTAM

\begin{tabular}{|c|c|c|c|c|c|c|}
\hline \multirow[t]{2}{*}{ Administration route } & \multirow[t]{2}{*}{ Treatment } & \multicolumn{3}{|l|}{ Oogram pattern } & \multicolumn{2}{|l|}{ EPG } \\
\hline & & Immature eggs $(\%)$ & Mature eggs (\%) & Dead ems $(\%)$ & Total & $\%$ Reduction \\
\hline \multirow[t]{2}{*}{ Intraperitoneal } & Control & $68.2 \pm 4.6$ & $26.2 \pm 4.1$ & $5.8 \pm 1.7$ & $1084 \pm 322$ & - \\
\hline & cTAM & $18.1 \pm 3.3^{*}$ & $70.5 \pm 2 *$ & $7.2 \pm 2.1$ & $214 \pm 53^{*}$ & $80 \%$ \\
\hline \multirow[t]{2}{*}{ Oral } & Control & $77.6 \pm 6$ & $18.4 \pm 5.1$ & $4 \pm 1.7$ & $1521 \pm 339$ & - \\
\hline & cTAM & $13 \pm 6.9 *$ & $66 \pm 9.6^{*}$ & $13.6 \pm 4.5$ & $181 \pm 91 *$ & $88 \%$ \\
\hline
\end{tabular}

*Significant reduction when compared with control group, $p<0.0001$

The original article has been corrected.

Publisher's note Springer Nature remains neutral with regard to jurisdictional claims in published maps and institutional affiliations. 